\title{
Improved ansatz for the direct correlation function in dilute nematic colloids
}

\author{
T.G.Sokolovska ${ }^{1,2}$, R.O.Sokolovskii ${ }^{1,2}$, G.N.Patey ${ }^{1}$ \\ ${ }^{1}$ Department of Chemistry, University of British Columbia, Vancouver, British Columbia, Canada V6T 1Z1 \\ 2 Institute for Condensed Matter Physics, 1 Svientsitskii Str., Lviv 79011, Ukraine
}

Received May 25, 2007, in final form September 4, 2007

\begin{abstract}
We present an improved analytical ansatz for the colloid-nematic direct correlation function. This ansatz is more accurate than our earlier version, and yields numerical results that are very close to the true mean spherical approximation for dilute nematic colloids. Furthermore, the improved ansatz is valid for external fields of any strength. We examine the zero-field behavior of the colloid-colloid potential of mean force in light of the improved ansatz, and show that at zero field and large separations this function decays as $R^{-5}$ and grows as $\Sigma^{6}$, where $\Sigma$ is the colloidal diameter. These dependencies are consistent with our earlier conclusions based on a less accurate version of the ansatz. As with the original ansatz, the improved version remains analytical and can be readily applied to a broad range of physically interesting systems. These include patterned and nonspherical colloids, colloids trapped at interfaces, and nematic fluids in confined geometries, e.g. in droplets.
\end{abstract}

Key words: colloid, liquid crystal, nematic, potential of the mean force

PACS: $61.30 . C z, 82.70 . D d, 61.30 . G d$

\section{Introduction to molecular modelling and correlations in nematic colloids}

Nematic colloids consist of colloidal particles suspended in nematic liquid crystal. These are of much current interest because the colloidal particles show a variety of structures that can be altered by varying physical factors such as the strength and direction of external fields [1,2]. Nematic colloids are intriguing from both fundamental and practical perspectives. All molecular interactions in the system are short-ranged, but colloidal particles effectively interact as oriented multipoles at long distances. In a way these systems are the inverse of fluids of charged particles, where the interaction potential is long-ranged but the correlations are short-ranged due to screening. We believe that the origin of such electrostatic-like behavior is the presence of long-range correlations in the bulk. In [3] we applied the Ornstein-Zernike (OZ) equation to nematic colloids to show how the electrostatic analogy asymptotically appears in the molecular theory of correlations. Our theory starts at the level of particle interactions modeled in a physically relevant way, and employs the Ornstein-Zernike and Lovett equations (OZL) for pair correlation functions and single-particle distributions. The general solution of these equations gives a description of long-range and shortrange effects in nematic colloids, and takes account of orientational and density fluctuations. The approach can be equally applied to concentrated and dilute mixtures for colloidal particles of any size ranging from nanometers to micrometers. After the solution is obtained, the corresponding thermodynamics is available via exact relations of statistical mechanics $[4,5]$.

The equilibrium single-particle distribution and pair correlation functions for nematic colloids are found by solving the OZ relationship,

$$
h_{\alpha \beta}(1,2)=c_{\alpha \beta}(1,2)+\sum_{\gamma=C, N} \int c_{\alpha \gamma}(1,3) \rho_{\gamma}(3) h_{\gamma \beta}(3,2) \mathrm{d}(3),
$$

combined with the exact Lovett equation [6]. In equation (1), $c_{\alpha \beta}$ and $h_{\alpha \beta}$ are the direct and total correlation functions, $\rho_{\mathrm{N}}(3), \rho_{\mathrm{C}}(3)$ are the density distributions of the nematic $(N)$ and 
colloidal $(C)$ components, and $\alpha, \beta$ represent $C$ or $N$. Also, for a nematogen, the label 1 denotes the coordinates $\left(\boldsymbol{R}_{1}, \hat{\boldsymbol{\omega}}_{1}\right)$, and for a spherical colloid $1 \equiv\left(\boldsymbol{R}_{1}\right)$. The functions in equation (1) describe all structural properties of the mixture at nanoscale and microscale levels. The exact OZ equation must be solved in conjunction with another approximate expression relating the total and direct correlation functions, such as the Percus-Yevick, hypernetted chain (HNC) or mean spherical approximation (MSA) closures. A closure is the only approximation that is necessary in the integral equation theory.

For a dilute $\left(\rho_{\mathrm{C}} \rightarrow 0\right)$ nematic colloid, the OZ equation takes the form,

$$
h_{\alpha \beta}(1,2)=c_{\alpha \beta}(1,2)+\int c_{\alpha N}(1,3) \rho_{\mathrm{N}}(3) h_{N \beta}(3,2) \mathrm{d}(3) .
$$

The physical meaning of all functions in equation $(2)$ is clear; $\rho_{\mathrm{N}}(\hat{\boldsymbol{\omega}})$ gives the orientational distribution in a pure nematic, whereas, $\rho_{\mathrm{N}}(1)\left[1+h^{\mathrm{NC}}(1,2)\right]$ gives the complete distribution of nematic fluid about a colloidal particle. The latter function takes into account all changes at a given point $\boldsymbol{R}_{1}$ induced by the colloidal particle at $\boldsymbol{R}_{2}$; these include changes in the local density and in the orientational distribution of the nematic fluid. The colloid-colloid potential of mean force at the HNC level is conveniently given by

$$
\phi^{\mathrm{CC}}(1,2)=\left[c^{\mathrm{CC}}(1,2)-h^{\mathrm{CC}}(1,2)\right] k_{\mathrm{B}} T+v^{\mathrm{CC}}(1,2),
$$

where $v^{\mathrm{CC}}(1,2)$ is the direct pair interaction between the colloidal particles.

We consider a model [7-9] consisting of a system of uniaxial particles [nematogens $(N)$ ] interacting through a pair potential taken to be the sum of a hard-sphere interaction (sphere diameter $\sigma$ ) and an anisotropic part defined by

$$
v(1,2)=v_{2}\left(R_{12}\right) P_{2}\left(\hat{\boldsymbol{\omega}}_{1} \cdot \hat{\boldsymbol{\omega}}_{2}\right)
$$

where $P_{2}\left(\hat{\boldsymbol{\omega}}_{1} \cdot \hat{\boldsymbol{\omega}}_{2}\right)$ is the second-order Legendre polynomial, $R_{12}$ is the center-center distance, the unit vector $\hat{\boldsymbol{\omega}}_{i}$ denotes the orientation of particle $i$, and

$$
v_{2}\left(R_{12}\right)=-A_{\mathrm{N}}\left(z_{\mathrm{N}} \sigma\right)^{2} \frac{\exp \left(-z_{\mathrm{N}} R_{12}\right)}{R_{12} / \sigma}
$$

Here, $A_{\mathrm{N}}$ and $z_{\mathrm{N}}$ are the energy and the length parameters characterizing the interaction. The nematogen interaction with the external field is given by

$$
v_{\mathrm{N}}(1)=-W \sqrt{5} P_{2}\left(\hat{\boldsymbol{\omega}}_{1} \cdot \hat{\boldsymbol{n}}\right), \quad W>0,
$$

where $W$ is the field strength. This interaction orders the bulk director $\hat{\boldsymbol{n}}$ parallel to the field. The OZL equations for this model have been solved analytically in the MSA $[7,8]$.

The model colloidal particles $(C)$ are taken to be hard spheres of diameter $\Sigma$. Van der Waals or other direct colloid-colloid interactions could easily be included through the $v^{\mathrm{CC}}(1,2)$ term in equation (3). The interaction of nematogens with the surface of a colloidal particle is modeled as

$$
v^{\mathrm{NC}}(1,2)=\left\{\begin{array}{l}
\infty, \quad \text { if } \quad s_{12}<\sigma / 2, \\
-A_{\mathrm{C}} \exp \left[-z_{\mathrm{C}}\left(s_{12}-\sigma / 2\right)\right] P_{2}\left(\hat{\boldsymbol{\omega}}_{1} \cdot \hat{\boldsymbol{s}}_{12}\right), \quad \text { if } \quad s_{12}>\sigma / 2,
\end{array}\right.
$$

where $s_{12}$ is a vector connecting the nearest point of the surface of colloid 2 with the center of nematogen 1 , and $\hat{\boldsymbol{s}}_{12}=s_{12} / s_{12}$. Note that positive and negative values of $A_{\mathrm{C}}$ favour, respectively, perpendicular and parallel orientations of nematogen molecules with respect to the surface. For $z_{\mathrm{C}} \sigma=1$ the nematogen-colloid interaction range is of the order of the nematogen "length". The strength of the nematogen-colloid interaction is determined by $A_{\mathrm{C}}$, and $z_{\mathrm{C}}$, and will vary for different surfactants. The magnitude of $A_{\mathrm{C}}$ depends on the surfactant concentration on the colloidal surfaces.

For micron and submicron colloids the solution of equation (2) can be greatly simplified for model (4-7). For this case we have suggested an ansatz, namely that the direct correlation function 
$c^{\mathrm{CN}}(1,2)$ can be taken from the wall-nematic solution that has been obtained for any orientation of the wall with respect to the field $[9,10]$. It is important that the solution was obtained subject to well defined boundary conditions at infinity: the director parallel to the field. This ansatz is very convenient because within the MSA the wall-nematic direct correlation function can be obtained in explicit form [11]. This permits explicit microscopic calculations of the density and orientational profiles [12], as well as effective interactions (potentials of mean force) between pairs of colloidal particles [13]. If the director $\hat{\boldsymbol{n}}$ is fixed parallel to the $z$-axis, the total correlation functions in the MSA are found in the form of spherical harmonic expansions,

$$
\begin{aligned}
h^{\mathrm{NN}}\left(R_{12}, \hat{\boldsymbol{\omega}}_{1}, \hat{\boldsymbol{\omega}}_{2}\right) & =\sum_{l l^{\prime}=0,2} \sum_{m} h_{l l^{\prime} m}^{\mathrm{NN}}\left(R_{12}\right) Y_{l m}\left(\hat{\boldsymbol{\omega}}_{1}\right) Y_{l^{\prime} m}^{*}\left(\hat{\boldsymbol{\omega}}_{2}\right), \\
h^{\mathrm{CN}}\left(\boldsymbol{R}_{12}, \hat{\boldsymbol{\omega}}_{2}\right) & =\sum_{l l^{\prime}=0,2} \sum_{m} h_{l l^{\prime} m}^{\mathrm{CN}}\left(R_{12}\right) Y_{l m}\left(\hat{\boldsymbol{R}}_{12}\right) Y_{l^{\prime} m}^{*}\left(\hat{\boldsymbol{\omega}}_{2}\right) .
\end{aligned}
$$

If all correlation lengths are finite and smaller than the colloidal radius, the ansatz gives a very good approximation. A problem arises if some correlation length is larger than $\Sigma$. The correlation length of $h_{22|m|=1}^{\mathrm{NN}}\left(R_{12}\right)$ in the nematic phase depends on the external field $W[3]$,

$$
\xi=\sqrt{\frac{K}{W \rho_{\mathrm{N}} S_{2} 3 \sqrt{5}}}
$$

where the elastic constant $K$ and order parameter $S_{2}$ are not sensitive to the influence of small fields. This means that in the case of zero field the correlation length is infinite. It was shown in [7] that these critical (Goldstone or elastic) correlations decrease at long distances as $1 / R_{12}$. Moreover, they generate long-range asymptotes in $h^{\mathrm{CN}}\left(\boldsymbol{R}_{12}, \hat{\boldsymbol{\omega}}_{2}\right)$ harmonics, in particular those that are coupled with $h_{22|m|=1}^{\mathrm{NN}}\left(R_{12}\right)$ through equation $(2)$. Physically this means that a single colloidal particle induces long-range distortions in the distribution of surrounding nematic. Using the ansatz for the colloid-nematic direct correlation function we obtain [3] the following asymptote for the total correlation function at zero field and $\Sigma \gg \sigma$,

$$
h^{\mathrm{CN}}(1,2) \stackrel{R \rightarrow \infty}{\longrightarrow} \frac{1}{10} \frac{h_{221}^{\mathrm{WN}}(s=\sigma / 2)}{B z_{\mathrm{C}}} \frac{\Sigma^{3}}{R^{3}}\left[Y_{21}\left(\hat{\boldsymbol{R}}_{12}\right) Y_{21}^{*}\left(\hat{\boldsymbol{\omega}}_{2}\right)+c . c .\right],
$$

where $h_{221}^{\mathrm{WN}}(s=\sigma / 2)$ is the contact value of the corresponding harmonic of the wall-nematic correlation function, and $B$ can be expressed through the bulk elastic constant $K$ and order parameter $S_{2}$,

$$
B^{2}=\frac{\left\langle\left|Y_{21}\right|^{2}\right\rangle_{\omega} \beta K}{15 \rho_{\mathrm{N}} S_{2}^{2}}
$$

The notation $\langle\ldots\rangle_{\omega}$ indicates $\int f_{\mathrm{N}}(\hat{\boldsymbol{\omega}})(\ldots) \mathrm{d} \hat{\boldsymbol{\omega}}$, and $f_{\mathrm{N}}\left(\hat{\boldsymbol{\omega}}_{1}\right)=\rho_{\mathrm{N}}(1) / \rho_{\mathrm{N}}$ is a single-particle distribution function in the bulk. Finally, at the HNC level the asymptote of the effective colloidal interaction was found in [3] to be

$$
\beta \phi^{C C^{\prime}}(\boldsymbol{R}) \stackrel{R \rightarrow \infty}{\longrightarrow} \frac{8 \pi}{15} \frac{h_{221}^{\mathrm{WN}}(s=\sigma / 2)}{z_{\mathrm{C}}} \frac{h_{221}^{W^{\prime} N}(s=\sigma / 2)}{z_{C^{\prime}}} \rho_{\mathrm{N}}\left\langle\left|Y_{21}\right|^{2}\right\rangle_{\omega} \frac{\Sigma^{3} \Sigma^{\prime 3}}{R^{5}} Y_{40}(\hat{\boldsymbol{R}}) .
$$

As mentioned above, the ansatz is reliable when $\xi<\Sigma$, but may give inaccurate results at small fields, where $\xi>\Sigma$. We investigated the reliability of the ansatz in [11]. The test calculations were based on verification of an exact consequence of the impenetrability of hard cores, namely $h^{\mathrm{CN}}\left(\boldsymbol{R}_{12}, \hat{\boldsymbol{\omega}}_{2}\right)=-1$ if $R_{12}<\frac{\Sigma+\sigma}{2}$. Thus, the exact condition implies that

$$
h_{220}^{\mathrm{CN}}\left(R_{12}\right)=h_{200}^{\mathrm{CN}}\left(R_{12}\right)=h_{020}^{\mathrm{CN}}\left(R_{12}\right)=h_{22 m}^{\mathrm{CN}}\left(R_{12}\right)=0, \quad h_{000}^{\mathrm{CN}}\left(R_{12}\right)=-1,
$$

for $R_{12}<(\Sigma+\sigma) / 2$. We found that since harmonics with $|m| \neq 1$ are not coupled with elastic bulk harmonics and are relatively short-ranged, for these harmonics our ansatz essentially satisfies 
the core condition for colloidal sizes larger than $50 \sigma$ for all system parameters considered. This is not the case for the harmonics $h_{22 \pm 1}^{\mathrm{CN}}\left(R_{12}\right)$. The problematic harmonics calculated with the ansatz are plotted in figure 1 for two colloid sizes, $\Sigma=50 \sigma$ and $500 \sigma$, and small, $W=10^{-4} k_{\mathrm{B}} T$, and zero external fields. The colloidal length parameter $z_{\mathrm{C}} \sigma=0.2$. The remaining model parameters are the same for all numerical calculations presented in this paper: $z_{\mathrm{N}} \sigma=1, A_{\mathrm{N}}=k_{\mathrm{B}} T, \eta=\pi \rho \sigma^{3} / 6=0.35$ and $A_{\mathrm{C}} / A_{\mathrm{N}}=2$. One can see that the condition (14) essentially holds at $W=10^{-4} k_{\mathrm{B}} T$ for the larger particle, but not for $\Sigma=50 \sigma$. Note that the correlation length equals $161.5 \sigma$ at the given field. In the zero field case, where $\xi$ is infinite, the core condition is not well satisfied for colloidal particles of any size.
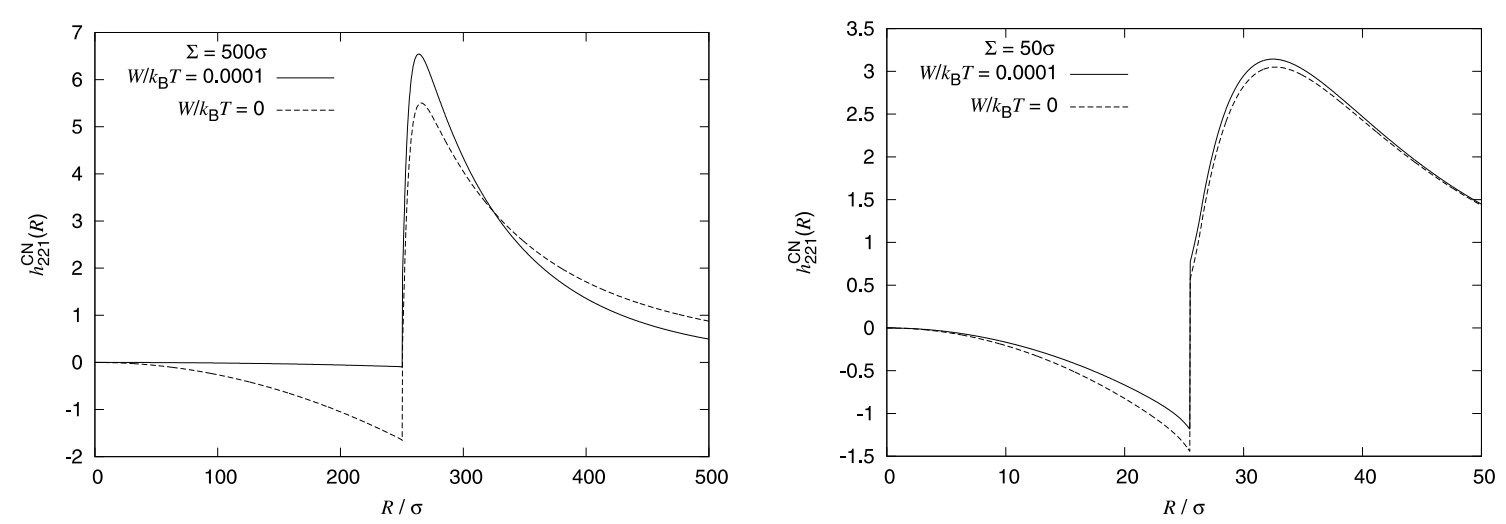

Figure 1. Long-range harmonics of the colloid-nematic total correlation function $h_{221}^{\mathrm{CN}}(R)$ for colloidal particles with $\Sigma=50 \sigma$ and $500 \sigma$, at zero external field and at $W=10^{-4} k_{\mathrm{B}} T$. The parameter $z_{\mathrm{C}} \sigma=0.2$.

Clearly our ansatz requires improvement in the low field (large $\xi$ ) limit, and this is the purpose of the present paper. We show how the ansatz can be much improved and examine the consequences for the zero-field asymptotes defined by equations (10) and (12).

\section{Corrected ansatz for small or zero fields}

Numerically, it is possible to correct the ansatz taken from the wall problem by introducing a single parameter. We scale the analytical expression for harmonics $|m|=1$ inside the hard core,

$$
\bar{c}_{221}^{\mathrm{CN}}\left(R_{12}\right) \approx q c_{221}^{\mathrm{WN}}\left(s=R_{12}-\Sigma / 2\right) \quad \text { for } \quad R_{12}<\frac{\Sigma+\sigma}{2}
$$

and choose the coefficient $q(0<q<1)$ such as to minimize

$$
\int_{0}^{(\Sigma+\sigma) / 2}\left|\bar{h}_{221}^{\mathrm{CN}}\left(R_{12}\right)\right| \mathrm{d} R_{12} .
$$

Note that $\bar{c}_{221}^{\mathrm{CN}}\left(R_{12}\right)$ outside the core remains the same and satisfies the MSA closure,

$$
c^{\mathrm{CN}}\left(\boldsymbol{R}_{12}, \hat{\boldsymbol{\omega}}_{2}\right)=-\frac{\phi^{\mathrm{CN}}\left(\boldsymbol{R}_{12}, \hat{\boldsymbol{\omega}}_{2}\right)}{k_{\mathrm{B}} T}, \quad \text { if } \quad R_{12}>\frac{\Sigma+\sigma}{2} .
$$

The corrected ansatz appears to be very close to the true MSA solution of equation (2), even at zero field (see figure 2 ). We have calculated $\bar{h}_{221}^{\mathrm{CN}}\left(R_{12}\right)$ with the corrected ansatz (15) for colloids with $\Sigma=500 \sigma$ and different surface parameters. At zero field $\bar{h}_{221}^{\mathrm{CN}}\left(R_{12}\right) / h_{221}^{\mathrm{CN}}\left(R_{12}\right) \approx 1.27$ for $z_{\mathrm{C}} \sigma=1$, and $\approx 1.23$ for $z_{\mathrm{C}} \sigma=0.2$ (figure 3 ). Numerically, we find that the deviation from $5 / 4$ lies within 2 per cent. Thus for sufficiently large colloidal particles, the asymptote (11) can be easily 

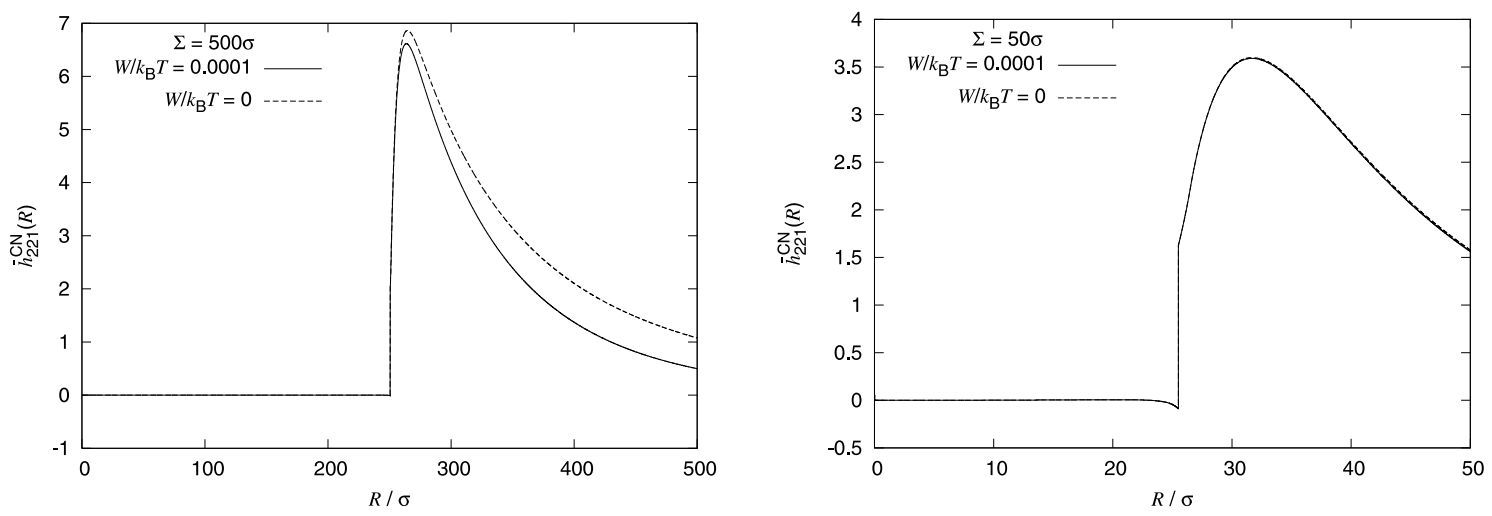

Figure 2. Corrected long-range harmonics of the colloid-nematic total correlation function $\bar{h}_{221}^{\mathrm{CN}}(R)$ for colloidal particles with $\Sigma=50 \sigma$ and $500 \sigma$, at zero external field and at $W=$ $10^{-4} k_{\mathrm{B}} T$. The parameter $z_{\mathrm{C}} \sigma=0.2$.

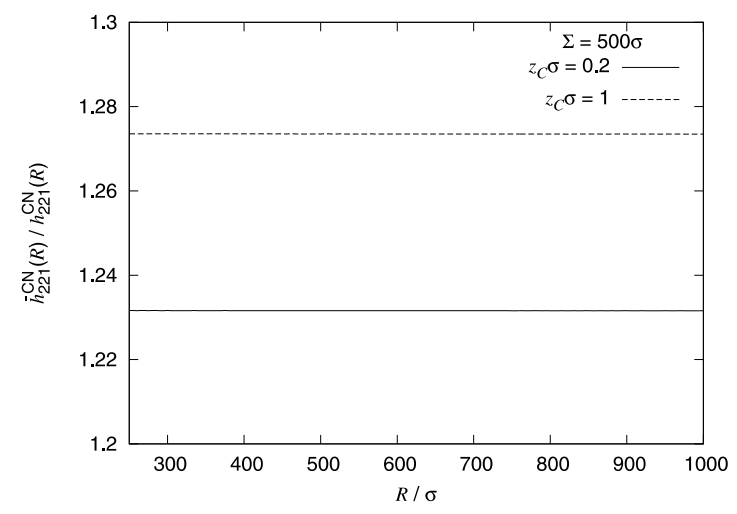

Figure 3. The ratio of the corrected long-range harmonics of the colloid-nematic total correlation function to the uncorrected case, $\bar{h}_{221}^{\mathrm{CN}}\left(R_{12}\right) / h_{221}^{\mathrm{CN}}\left(R_{12}\right)$, at zero external field, for $\Sigma=500 \sigma$ and for two ranges of the colloid-nematic interaction, $z_{\mathrm{C}} \sigma=1$ and $z_{\mathrm{C}} \sigma=0.2$.

corrected at zero field by including a factor of 5/4. In experiments typical colloidal sizes are of order $10^{3} \sigma$.

The factor $5 / 4$ is not accidental. Formally we can take the limit of zero field $(\xi \rightarrow \infty)$ for the case of the tilted wall (from equation (17) of [11]). In this case the asymptote of the wall-nematogen total correlation function tends to a constant value at large distances from the wall,

$$
\lim _{\xi \rightarrow \infty} h^{\mathrm{WN}}\left(\boldsymbol{s}, \hat{\boldsymbol{\omega}}_{\mathrm{N}}\right) \stackrel{s \rightarrow \infty}{\longrightarrow} \lim _{\xi \rightarrow \infty} \frac{h_{221}^{\mathrm{WN}}(s=\sigma / 2)}{B z_{\mathrm{C}}}\left[Y_{21}(\hat{\boldsymbol{s}}) Y_{21}^{*}\left(\hat{\boldsymbol{\omega}}_{\mathrm{N}}\right)+\text { c.c. }\right] \exp [(-s-\sigma / 2) / \xi],
$$

where the unit vector $\hat{s}$ defines the orientation of the wall normal with respect to the field, and $\hat{\omega}_{\mathrm{N}}$ is a molecule orientation.

On the other hand, we can consider the limit of the distribution function around a spherical colloid [equation (10)] as $\Sigma \rightarrow \infty$. Note that the limit of zero field, $\xi \rightarrow \infty$, is taken first. At the distance $s=R-\Sigma / 2$ outside the surface, $\Sigma^{3} / R^{3}$ tends to 8 , since $\Sigma \rightarrow \infty$ and $s \ll \Sigma$. By putting $\hat{\boldsymbol{s}} \equiv \hat{\boldsymbol{R}}_{12}$ and $\hat{\boldsymbol{\omega}}_{\mathrm{N}} \equiv \hat{\boldsymbol{\omega}}_{2}$ one can see from equation (11) that

$$
\lim _{\xi \rightarrow \infty} h_{221}^{\mathrm{WN}}(s)=\frac{5}{4} \lim _{\Sigma \rightarrow \infty} \lim _{\xi \rightarrow \infty} h_{221}^{\mathrm{CN}}(s) .
$$

In other words, the order in which two limits are taken does matter if one uses the direct correlation function from the wall problem as an ansatz for spherical colloids. If the asymptote is corrected with 
a factor of 5/4 the inconsistency does not appear. Following [3], one can see that any correction of asymptote (10) with a given factor results in a correction of asymptote (12) with the same factor squared. Thus, the asymptote of the potential of mean force should be corrected with a factor of $(5 / 4)^{2}$.

If the correlation length $\xi$ is smaller than $\Sigma$, one would expect the fluid distribution about a colloid obtained with the ansatz to coincide with the distribution near the plane wall, at least for $s \ll \Sigma$. Our numerical analysis for $\Sigma=500 \sigma, z_{c} \sigma=1$ and distances up to several $10 \sigma$ shows that this is true for $\beta W=10^{-4}$ and $\xi=161.5 \sigma$. The picture is different, however, for the smaller field, $\beta W=10^{-6}$, where $\xi \sim 1615 \sigma$ is larger than $\Sigma$. In this case $h_{221}^{\mathrm{WN}}(s) / h_{221}^{\mathrm{CN}}(s)$ tends to $5 / 4$ at $s \approx 10 \sigma$.

We are especially interested in verifying the $\Sigma$-dependencies of the asymptotes (10) and (12), particularly because phenomenological theories appear to disagree on this matter. A theory with imposed weak boundary conditions at colloidal surfaces predicts the interaction of colloids (that exhibit up-down symmetry) to be proportional to $\Sigma^{8}[14]$. At the same time the theory of Lubensky et al. [15], based on rigid boundary conditions, predicts that the interactions behave as $\Sigma^{6}$. Our molecular theory does not impose boundary conditions at colloidal surfaces, but instead calculates them. All information about any $\Sigma$-dependence is contained in the direct colloid-nematic correlation function. In fact the $\Sigma$-dependence of asymptote (10) at zero field is defined by the $c_{221}^{\mathrm{CN}}$ behavior at small $k$ in Fourier space. The uncorrected ansatz gives the following expansion for large $\Sigma$ at zero field [3],

$$
c_{221}^{\mathrm{CN}}(k) \stackrel{k \rightarrow 0}{\longrightarrow}-4 \pi \frac{h_{221}^{\mathrm{WN}}(s=\sigma / 2)}{30 z_{\mathrm{C}}} B\left[\Sigma^{3}+O\left(\Sigma^{2}\right)\right] k^{2}+O\left(k^{4}\right) .
$$

To investigate further, we consider the expansion (20) for the corrected ansatz, which takes the form,

$$
\bar{c}_{221}^{\mathrm{CN}}(k) \stackrel{k \rightarrow 0}{\longrightarrow}\left[4 \pi \frac{\beta A_{\mathrm{C}}(-1+q)}{75 z_{\mathrm{C}}}(\Sigma / 2)^{4}+O\left(\Sigma^{3}\right)\right] k^{2}+O\left(k^{4}\right) .
$$

The first impression is that the coefficient of $k^{2}$ now has a $\Sigma^{4}$-dependence. However, this turns out not to be true. Numerical calculations of $(-1+q) \Sigma$ were done for different $\Sigma$, up to $1500 \sigma$. For $\sigma=2 n m$, order of size of $5 \mathrm{CB}$ molecules, this corresponds to colloids of $3 \mu \mathrm{m}$ in diameter. We considered various interaction parameters $\left(z_{\mathrm{C}} \sigma=0.1,0.2,1,2\right)$. In all cases the value of $(-1+q) \Sigma$ tends to a constant limit at large $\Sigma$ (figure 4 ). In other words, the fact that $q \neq 1$ corrects asymptotes (10) and (12) with the factors $5 / 4$ and $(5 / 4)^{2}$, respectively, but does not change their $\Sigma$-dependence at zero field. Thus, the MSA results support the conclusion on the $\Sigma$-dependence reached in [15], where rigid boundary conditions were employed. It should be emphasized that in our model the calculated director distribution in the vicinity of colloids is not coupled rigidly with normals to the colloidal surface [12]. The full coefficient of $k^{2}$ in equation (21) divided by $\Sigma^{3} / z_{\mathrm{C}}$ is plotted in figure 5 for different values of $z_{\mathrm{C}} \sigma$. One can see that a $\Sigma^{3}$-dependence holds in all the cases for $\Sigma>500 \sigma$.

\section{Summary and conclusions}

In this paper we have developed an improved ansatz for the colloid-nematogen direct correlation function. In our previous studies we suggested that a general solution of the Ornstein-Zernike equation for a nematic fluid in the presence of a wall, arbitrarily oriented with respect to the nematic director, can be very useful for the investigation of dilute nematic colloids. In fact, we suggested using the wall-nematogen direct correlation function, obtained explicitly in [11], as an ansatz for the direct correlation function in nematic colloids. This ansatz permits explicit microscopic calculations of the density and orientational profiles, as well as potentials of mean force for large colloidal particles, such as those used in numerous experiments. This ansatz works very well in the presence of external fields, when all correlation lengths are smaller than the colloidal radius. At small and zero external fields the results of this ansatz, particularly asymptotes at zero field, need some correction. The reason for this is that the "elastic" correlation length in the nematic phase is infinite at zero field. Here we suggest a simple scaling of the ansatz, which gives results 


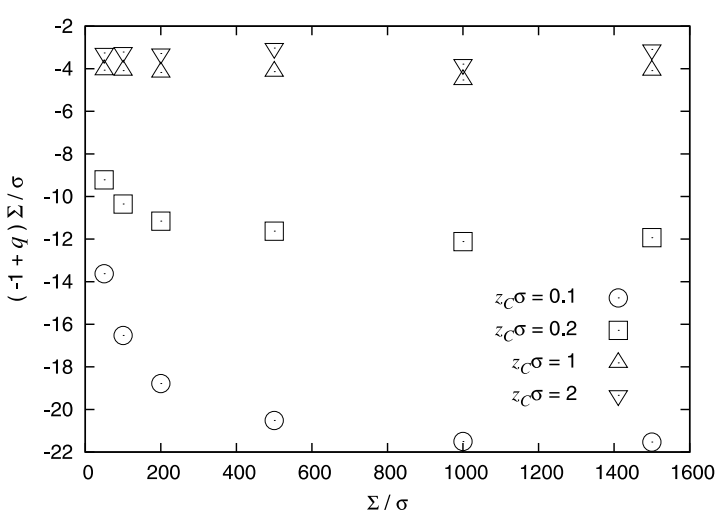

Figure 4. The values of $(-1+q) \Sigma$ for various ranges of the colloid-nematic interaction, $z_{\mathrm{C}} \sigma=0.1,0.2,1,2$.

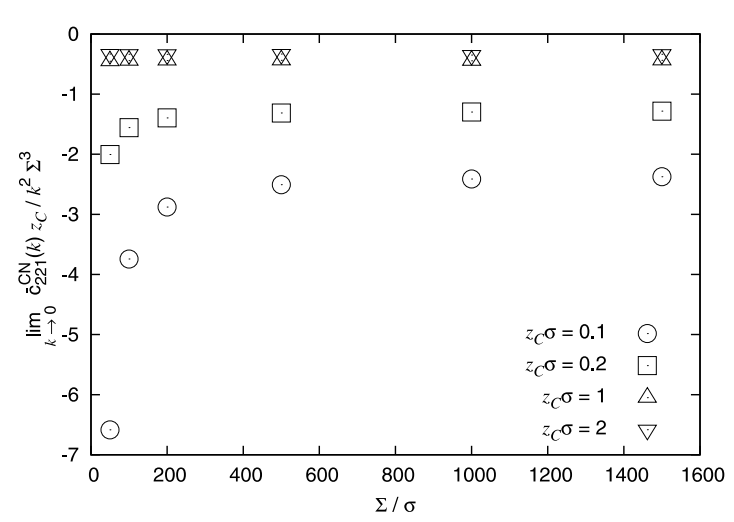

Figure 5. The $\lim _{k \rightarrow 0} \bar{c}_{221}^{\mathrm{CN}}(k) z_{\mathrm{C}} /\left(k^{2} \Sigma^{3}\right)$ at zero external field as a function of $\Sigma$ for various ranges of the colloid-nematic interaction $z_{\mathrm{C}} \sigma$.

numerically very close to the MSA solution of the OZ equation for nematic colloids in the presence of arbitrarily weak fields, including the zero field case. Our study of spherical colloids shows that the improved ansatz corrects asymptotes of the nematic distribution about a colloidal particle and the colloid-colloid potential of mean force with factors $5 / 4$ and $(5 / 4)^{2}$, respectively, but it does not change the dependence of the asymptotes on the colloidal diameter, or on the interparticle vector.

Finally, the ansatz taken from the wall-nematic problem has some additional advantages. Unlike a direct solution of the $\mathrm{OZ}$ equation for nematic colloids, the same analytical expression can be used with little adjustments for physically different systems, e.g., nonspherical colloids, colloids with a nonuniform distribution of surface surfactant (patterned colloids), colloids trapped at interfaces, as well as nematic droplets.

\section{Acknowledgements}

Acknowledge is made to the Donors of the American Chemical Society Petroleum Research Fund for partial support of this research. The financial support of the Natural Science and Engineering Research Council of Canada is gratefully acknowledged. This research has been enabled by the use of WestGrid computing resources, which are funded in part by the Canada Foundation for Innovation, Alberta Innovation and Science, BC Advanced Education, and the participating research institutions. WestGrid equipment is provided by IBM, Hewlett Packard and SGI.

\section{References}

1. Poulin P., Stark H., Lubensky T.C., Weitz D.A. Science, 1997, 275, 1770.

2. Smalyukh I.I., Lavrentovich O.D., Kuzmin A.N., Kachynski A.V., Prasad P.N. Phys. Rev. Lett., 2005, 95, 157801.

3. Sokolovska T.G., Sokolovskii R.O., Patey G.N., submitted to Phys. Rev. E.

4. Hansen J.P., McDonald I.R. Theory of Simple Liquids, 2nd ed. Academic, London, 1986.

5. Poniewierski A., Stecki J., Mol. Phys., 1979, 38, 1931.

6. Rowlinson J.S., Widom B. Molecular theory of capillarity. The international series of monographs on chemistry. Clarendon press, Oxford, 1989.

7. Holovko M.F., Sokolovska T.G., J. Mol. Liq., 1999, 82, 161.

8. Sokolovska T.G., Sokolovskii R.O., Holovko M.F. Phys. Rev. E, 2001, 64, 051710.

9. Sokolovska T.G., Sokolovskii R.O., Patey G.N., Phys. Rev. Lett., 2004, 92, 185508.

10. Sokolovska T.G., Sokolovskii R.O., Patey G.N., J. Chem. Phys., 2005, 122, 03470.

11. Sokolovska T.G., Sokolovskii R.O., Patey G.N., J. Chem. Phys., 2006, 125, 034903.

12. Sokolovska T.G., Sokolovskii R.O., Patey G.N., J. Chem. Phys., 2005, 122, 124907. 
13. Sokolovska T.G., Sokolovskii R.O., Patey G.N., Phys. Rev. E, 2006, 73, R020701.

14. Ruhwandl R.W., Terentjev E.M., Phys. Rev. E, 1997, 56, 5561.

15. Lubensky T.C., Pettey D., Currier N., Stark H., Phys. Rev. E, 1998, 57, 610.

\title{
Покращений анзац для прямої кореляційної функції в розведених нематичних колоїдах
}

\author{
Т.Г. Соколовська ${ }^{1,2}$, Р.О.Соколовський ${ }^{1,2}$, Г.Н.Пейті ${ }^{1}$ \\ 1 Хімічний факультет, Університет Британської Кулумбії, Ванкувер, Канада \\ 2 Інститут фізики конденсованих систем НАН України, вул. Свєнціцького 1, Львів 79011, Україна
}

Отримано 25 травня 2007 р., в остаточному вигляді - 4 вересня 2007 р.

\begin{abstract}
Ми представляємо покращений аналітичний анзац для прямої кореляційної функції колоїд-нематик. Цей анзац є точнішим ніж його попередня версія, отримана нами, і дає числові результати для розведених нематичних колоїдів, що є дуже близькими до тих, які отримуються в рамках справжнього середньо-сферичного наближення. Крім того, покращений анзац є справедливим для випадку зовнішніх полів довільної сили. Ми вивчаємо поведінку потенціалу середньої сили колоїд-колоїд в нульовому полі, використовуючи покращений анзац і показуємо, що в нульовому полі і на великих віддалях ця функція загасає як $R^{-5}$ і росте як $\Sigma^{6}$, де $\Sigma €$ діаметром колоїда. Ця залежність узгоджується з нашими попередніми обчисленнями, які базуються на менш точній версії анзацу. Як i в попередньому випадку, покращена версія залишається аналітичною і може бути легко застосована до широкого класу фізично цікавих систем, які включають колоїди з довільним розподілом сурфактантів на поверхні і несферичні колоїди, колоїди при міжфазній границі і нематичні плини в обмежених геометріях, наприклад в краплинах.
\end{abstract}

Ключові слова: колоїд, рідкий кристал, нематик, потенціал середньої сили

PACS: $61.30 . C z, 82.70 . D d, 61.30 . G d$ 